\title{
Optimization of IMU Indoor Localization with Wireless Sensors
}

\author{
Zhengyu $\mathrm{Yu}^{*}$ \\ Faculty of Engineering and \\ Information Technology \\ University of Technology, Sydney \\ Sydney, Australia \\ Email:Zheng.y.yu@ hotmail.com
}

\author{
Zenon Chaczko \\ Faculty of Engineering and \\ Information Technology \\ University of Technology, Sydney \\ Sydney, Australia \\ Email: Zenon.Chaczko@uts.edu.au
}

\begin{abstract}
This paper presents the development process of a wireless inertial measurement unit (IMU). A wireless communication configuration is designed for this unit. Low cost micro-electro-mechanical-systems (MEMS) accelerometers are used in the hardware prototyping.
\end{abstract}

Keywords-component; IMU; INS; accelerometers; MEMS; wireless communication, gyro-free

\section{INTRODUCTION}

An inertial measurement unit (IMU) provides selfcontained high frequency information for vehicle positioning systems. When external position signals are unavailable, the IMU can provide continuous vehicle positioning information. An IMU contains accelerometers and gyros. Accelerometers provide acceleration information, and gyros measure the rotation rate of a moving vehicle. An inertial navigation system (INS) can be built with three accelerometers, three gyros and associated positioning computing algorithms. The INS computes the vehicle positioning information using acceleration. By integration of the acceleration, the velocity of a vehicle is obtained, and the vehicle position is then computed by integration of the velocity. The vehicle accelerations and accelerometer measurements are in different moving coordinate systems. During this real time integration process, gyro measurements continuously provide vehicle orientation information to transfer the acceleration in different moving coordinate systems. Inertial sensors have been applied in bridges, dynamometers, and aircraft since the early 1920s, but high accuracy inertial measurement units have been expensive until recently. In the previous 20 years, INSs have been used in air vehicles, land vehicles, ocean vessels, and robots. In recent years, the reduction in the cost of inertial sensors has made INS positioning applications available to many other areas such as autonomous systems in agriculture, mining, robotics, transport, health care, pedestrian navigation, and smart phone applications. Wireless technologies and wireless sensor networks have now also emerged. Remote monitoring systems using wireless sensors have become a new research and development direction, and these wireless applications include robot positioning in disaster field discovery, healthcare monitoring systems, and so on. Consequently, there is a new need to develop wireless inertial sensors and understand the impacts of wireless IMUs for these positioning applications. The positioning mechanism using inertial sensors are based on the acceleration and velocity integration process. Any errors in acceleration will be integrated and amplified after integration. This thesis develops a wireless IMU which allows the IMU to communicate with a computer remotely and synchronously. An error model is developed for the wireless IMU to improve the accuracy of the system. The approach for modelling wireless inertial sensor errors is demonstrated in this work. The design and modelling approach are validated using dynamic positioning experiments in a gyro-free INS system.

\section{LITERATURE RESEARCH}

\section{A. MEMS accelerometer technology}

Inertial navigation systems have been commonly used by mechanical instruments for the measurement of a specific force in spring systems and mass accelerometers [1]. The full independent navigation measurement of specific force normally uses three single- axis accelerometers as well as multi-axis instruments. It is very common but not essential to setup up these three accelerometers with their three sensitive axes mutually orthogonal [1].

The following calibration parameters present a sensor linear model of a typical low cost MEMS sensor [2].

Bias of sensor X-axis $\left(B_{x}\right)$

Bias of sensor $\mathrm{Y}$-axis $\left({ }^{B}\right.$ )

Bias of sensor Z-axis $\left(B_{z}\right)$

Scale factor of sensor X-axis to X-axis inputs $\left(S_{x}\right)$

Scale factor of sensor Y-axis to Y-axis inputs $\left(S_{y}\right)$

Scale factor of sensor Z-axis to Z-axis inputs $\left(S_{z}\right)$ 


$$
\begin{aligned}
& \text { Misalignment of sensor X-axis to Y-axis inputs }\left(M_{x y}\right) \\
& \text { Misalignment of sensor X-axis to Z-axis inputs }\left(M_{x z}\right) \\
& \text { Misalignment of sensor Y-axis to Z-axis inputs }\left(M_{y z}\right) \\
& \text { Misalignment of sensor Z-axis to X-axis inputs }\left(M_{z x}\right) \\
& \text { Misalignment of sensor Z-axis to Y-axis inputs }\left(M_{z y}\right)
\end{aligned}
$$

\section{B. Implementation of inertial sensors}

In the experiments, the real bias drift of the accelerometer was $2.5 \mathrm{mg}$, whereas the error of the accumulated distance was $1 \mathrm{~cm}$. A low cost micro machined accelerometer, ADXL 202 produced by analog devices, was been used in these experiments [3]. The range of the measurement is $2 \mathrm{~g}$. Both static acceleration and dynamic acceleration can be measured. The system bandwidth is from $0.01 \mathrm{~Hz}$ to $5000 \mathrm{~Hz}$. A $50 \mathrm{~Hz}$ bandwidth and $200 \mathrm{~Hz}$ sampling rate were implemented in the experiment [3]. It should be noted that when the higher bandwidth was used, a higher level of white noise occurred. A robot arm was used in the experiments. 23 data sets with different velocity and acceleration were generated [3] and were separated into three different sets: low acceleration, moderately high acceleration and high acceleration. The final results showed that the error of integrated distance was $1.55 \mathrm{~cm}$ when the acceleration was $10 \mathrm{~m} / \mathrm{s}^{2}$. The higher the acceleration used, the lower were the random biases produced [3].

Barshan and Durant-Whyte [4] developed an error model of a low-cost inertial navigation system which was presented with an extended Kalman filter for estimating the acceleration, distance and orientation of mobile robots. Generally, IMU should be mounted on other navigation sensors to provide a precise vehicle location. Basically, accelerometers generate data of acceleration, whereas gyroscopes generate data of angular rate [4]. These data must be integrated to calculate the measurement of velocity, distance and orientation. Over a long period, a small error in the data could cause a big difference in distance. One method to deal with this issue is to add a navigation sensor which is able to correct the inertial sensor accumulated errors [4]. Through a detailed understanding of system drift errors, another method of overcoming errors is to use a priori information of the errors of the inertial sensors in an extended Kalman filter to provide the rate of the location prior to generating the data from INS sensing [4].

\section{Accelerometer sensor errors}

Every type of accelerometer contains errors which affect the accuracy of the measurement of the specific acting force. This type of accelerometer is able to produce very high performance with low biases, good linearity and with a dynamic range from $10^{4}$ to $10^{5}$. Following is a list of the major source of error discovered in mechanical accelerometers, as noted by Titterton [5]:

Fixed bias error is a bias or a displacement from zero according to the measurement of the acting force which is represented when the corresponding acceleration is zero [5]. The error arises due to the residual spring torques. The bias error is not dependent on any motion to which the accelerometer can be subjected. This error is normally represented in units from milli-g to micro-g, with the result depending on the precision of the instrument [5].

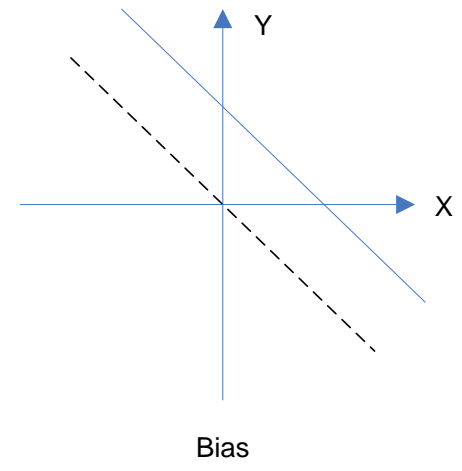

Figure 1. Bias Error

Scale factor errors are the errors of a ratio change between the output sensors and the input acceleration measurement. Error arises due to the effects of the temperature and the nonideal performance of the device. Usually, the scale factor is represented as a percentage of the full scale quantity measurement or as a ratio, such as, parts per million (PPM). The nonlinearity scale-factor depends on the systematic deviations from the least-squares straight line, which refers to the implemented acceleration and the output signal [5].

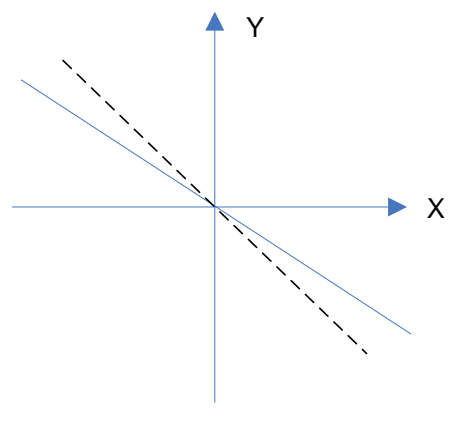

Scale Factor

Figure 2. Scale Factor Error

\section{Accelerometer Calibrator}

Lueck and Wolk [6] developed a quick method for calibrating accelerometers. The device and implementation of high performance accelerometers were introduced and the consideration of the errors was established. With linear equipment, it is easy to calibrate the outputs of the devices by the careful alignment of their sensitive axis according to the direction of gravity. The alignment accuracies within a few degrees are usually $0.1 \%$ and better. The method is presented 
without any alignment with gravity. The method of developing the sensor's performance is to remove structural errors from the sensor output. The difference between a sensor's output and the output of its measurement is defined as a structural error, which occurs consistently when a new measurement has been taken. Errors can be calculated and removed digitally in real-time by using the actual output of measurements during calibrations. Calibration is used to improve the accuracy of the sensors [6].

Four angles calibration:

The equation for position 1 is below:

$$
N_{1}=a g \sin (\theta)+b
$$

$a$ stands for the sensitivity of the accelerometer. $b$ stands for the offset and bias of the accelerometer. $\theta_{\text {stands for the }}$ unknown angle between the local vertical gravity and the accelerometer axis. Both $a$ and $b$ can be determined during the calibration, therefore the output $N$ can be presented in terms of $g$ of acceleration [6].

The equation for positions 2, 3 and 4 are expressed as:

$$
\begin{aligned}
& N_{2}=a g \sin \left(\theta+\frac{\pi}{2}\right)+b \\
& N_{3}=a g \sin (\theta+\pi)+b \\
& N_{4}=a g \sin \left(\theta-\frac{\pi}{2}\right)+b
\end{aligned}
$$

From symmetry and trigonometry the equations can be solved without knowing $\theta$ :

$$
\begin{aligned}
& \sin (\theta+\pi)=-\sin \theta \\
& \sin \left(\theta+\frac{\pi}{2}\right)=-\sin \left(\theta-\frac{\pi}{2}\right)=\cos (\theta)
\end{aligned}
$$

Therefore,

$$
\begin{aligned}
& b=\frac{1}{4}\left\{N_{1}+N_{2}+N_{3}+N_{4}\right\} \\
& a g=\frac{1}{2} \sqrt{\left(N_{1}-N_{3}\right)^{2}+\left(N_{2}-N_{4}\right)^{2}}
\end{aligned}
$$

Three angles calibration:

Three angles, equally reading 120 degrees can be used to calibrate the accelerometers [6].

The three equations are:

$$
\begin{aligned}
& N_{1}=a g \sin (\theta)+b \\
& N_{2}=a g \sin \left(\theta+\frac{2 \pi}{3}\right)+b=a g \cos \left(\theta+\frac{\pi}{6}\right)+b \\
& N_{3}=a g \sin \left(\theta-\frac{2 \pi}{3}\right)+b=a g \cos \left(\theta-\frac{\pi}{6}\right)+b
\end{aligned}
$$

The solution is

$$
\begin{aligned}
& b=\frac{1}{3}\left\{N_{1}+N_{2}+N_{3}\right\} \\
& a g=\frac{2}{3} \sqrt{N_{1}{ }^{2}+N_{2}{ }^{2}+N_{3}{ }^{2}-\left(N_{1} N_{2}+N_{1} N_{3}+N_{2} N_{3}\right)}
\end{aligned}
$$

\section{E. Multi-sensor IMU}

Wahyudi introduced a multi-sensor IMU system for a land vehicle in a range of motion in order for collecting the data from optimized sensitive sensors [7]. Liu presented a camera based IMU system to calibrate the parameters of estimations on V-SLAM technology [8]. Yijun designed a digital protractor based IMU to measure the attitude of two separated objects [9]. Benzerrouk integrated IMU and UWB to develop the optimization of the Pedestrian navigation [10].

\section{SYSTEM DESIGN}

A new wireless accelerometer communication design is presented. Currently, the majority of IMU accelerometers are designed with cable for data transmission. Some have been developed with wireless communications; however, the errors in such systems are significantly noticeable. In addition, the wireless communication device has been usually been fixed on the accelerometer and is not functional when implemented on other accelerometers. The following system enables these issues to be solved. A wireless communication mounted accelerometer has been designed, and a method of error reduction has been developed.
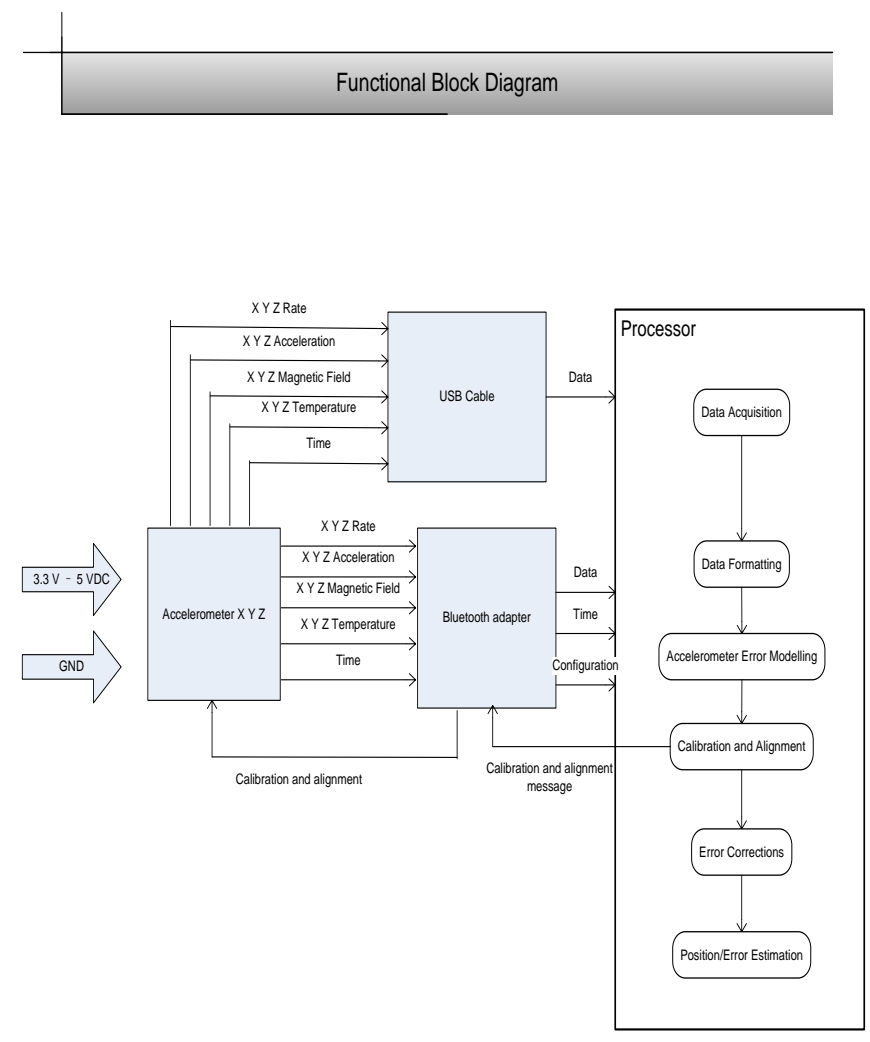

Figure 3. Functional Block Diagram

The wireless inertial measurement unit is designed as in Figure 3-1. The sensors of accelerometer (X, Y, Z) are set up in three orthogonal axes. By using Bluetooth adapter, Data is transferred to the computer attaching data of $\mathrm{X}, \mathrm{Y}, \mathrm{Z}$ accelerations, magnetic field, time and temperature. The baud 
rates on both the Bluetooth adapter and the computer have been adjusted to the same. When the date of the acceleration transfers, the computer calculates raw data into readable data which shows on the screen synchronously. Error modelling is developed to estimate and limited errors from wireless transmission by comparing the data from wireless communication with the data from the same device via cable communication. In the error modelling progress, a USB cable is connected to the computer in order for transferring the data by different communication methods. During the process, Data errors are addressed, so that algorithms and error modelling are developed to calibrate and align the data from the system. The calibration and alignment messages will be transferred back to the accelerators. The acceleration settings are adjustable during the process, and the updated data will again be sent to the computer via wireless communication. When the errors have been detected and reduced, the data will be implemented to determine the position of gyro-free INS.

\section{WIRELESS ACCELEROMETER UNIT ERROR MODELLING}

The specific forces on the three accelerometers in these six positions.

Accelerometer measurements are collected in the six positions. Accelerometer outputs measured in the six positions are compared with the specific forces.

From the experiments, it was found that the cross-axis errors make little contribution to the overall errors. The crossaxis error coefficients Bx_y, Bx_z, By_x, By_z, Bz_x, Bz_y can therefore be ignored during the modelling process. The accelerometer error model can be simplified as following:

$$
\begin{aligned}
& \delta f_{x}=b_{x}+S_{f_{-} x} \times a_{x}+m_{y_{-} x} \times a_{y}+m_{z_{-} x} \times a_{z}+\eta_{x} \\
& \delta f_{y}=b_{y}+S_{f_{-} y} \times a_{y}+m_{x_{-} y} \times a_{x}+m_{z_{-} y} \times a_{z}+\eta_{y} \\
& \delta f_{z}=b_{z}+S_{f_{-} z} \times a_{z}+m_{x_{-} z} \times a_{x}+m_{y_{-} z} \times a_{y}+\eta_{z}
\end{aligned}
$$

Model parameters are calculated using the following approach.

Position 5:

$$
\begin{aligned}
& b_{x}+m_{y_{-} x} \times(g)+\eta_{x}=\tilde{a}_{x}(5) \\
& b_{y}+S_{f_{-} y} \times(g)+\eta_{y}=\tilde{a}_{y}(5)-g \\
& b_{z}+m_{y_{-} z} \times(g)+\eta_{z}=-\tilde{a}_{z}(5)
\end{aligned}
$$

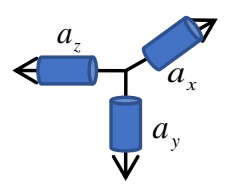

Figure 4. Position 5 System Position

$$
\begin{gathered}
a_{x}(5)=0 \\
a_{y}(5)=g \\
a_{z}(5)=0 \\
b_{x}+m_{y_{-} x} \times(g)+\eta_{x}=\tilde{a}_{x}(5) \\
b_{y}+S_{f_{-} y} \times(g)+\eta_{y}=\tilde{a}_{y}(5)-g \\
b_{z}+m_{y_{-} z} \times(g)+\eta_{z}=-\tilde{a}_{z}(5)
\end{gathered}
$$

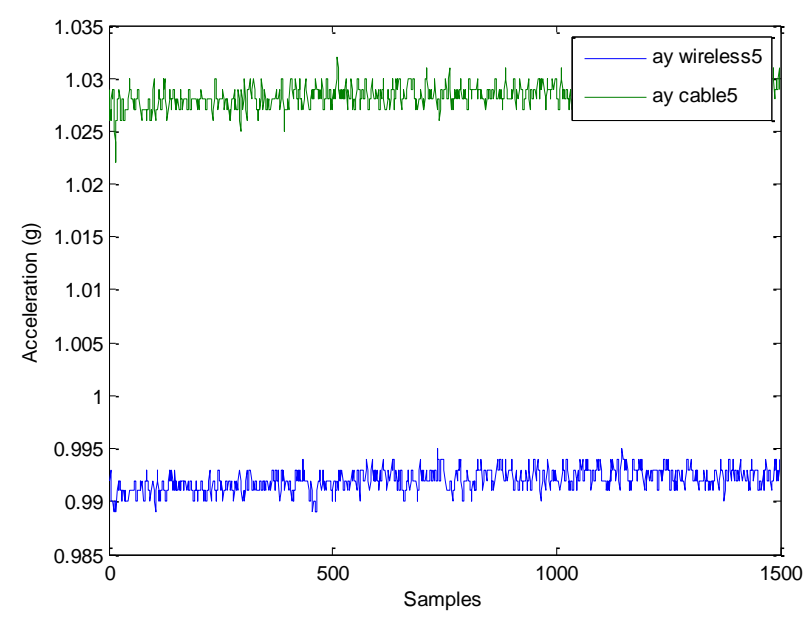

Figure 5. Position 5 Raw Data of Accelerations in Wireless and Cable Transmission

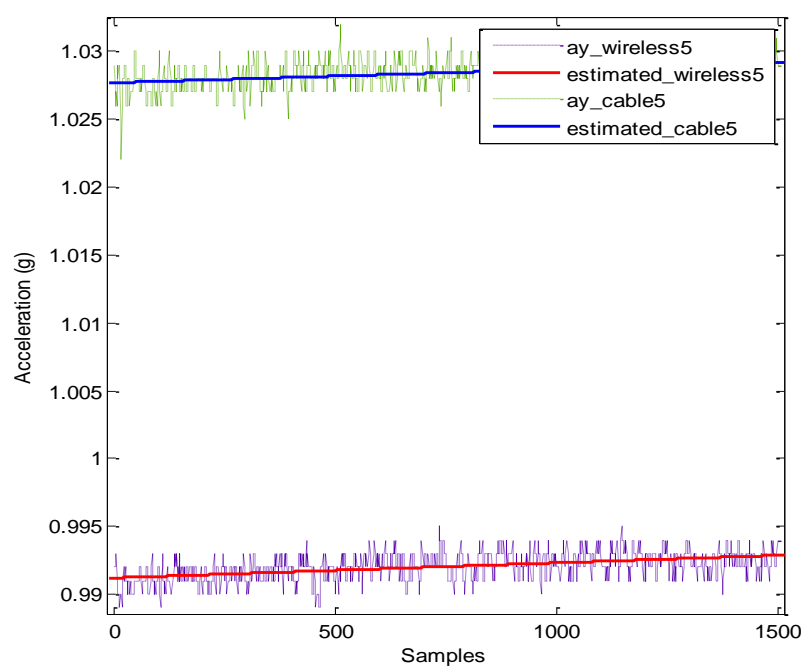

Figure 6. Position 5 Accelerations in Wireless and Cable

Transmission in Linear Model

In this figure, both acceleration data from wireless and cable transmission on axis $\mathrm{Y}$ have been presented as two lines As showed, there is a gap between those data, errors from bias and scale factors are not reduced before the error modelling. 


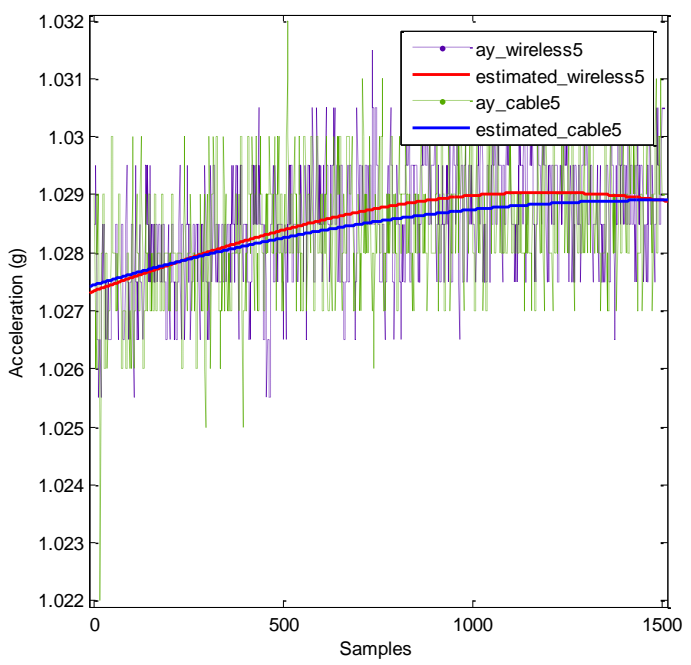

Figure 7. Position 5 Evolution of Estimated Errors

In this figure, both acceleration data from wireless and cable transmission on axis $\mathrm{Y}$ have been presented as two lines which have been developed by error modelling. As showed, they are very close, errors from bias and scale factors are reduced.

The errors from sensors are generated by the sensor device itself which is IMU in this case. The IMU previously introduced has been developed in several calibration experiments. The results of calibration show that the errors of cross-axis and quadratic coefficient do not change significantly in the lab, which developed the calibration of the accelerometer before the using of navigation system. Therefore, This system focus on the method of how to determine and reduce the bias and scale factor errors between wireless communications and cable transmission.

\section{RESULT AND FUTURE RESEARCH}

From the results, errors have been determined. The error modelling method is finally developed to estimate and correct a group of data from the experiments. The data from the cable is determined as the original data. The data from wireless communication has been determined as the estimated data which is needed to be developed. By using designed error modelling in the paper, it is clear to detect and reduce the errors from wireless transmission and any other transmission in the future. Nevertheless, the error modelling has been validated to be implemented in any INS. More developments in INS error modelling will be introduced in the future research.

\section{REFERENCES}

[1] Titterton, D.H.D.H. 2004a, 'Accelerometer and multi-sensor technology', in D. Titterton \& Weston, Eds., Strapdown Inertial Navigation Technology, 2nd, edn., vol. 5, Stevenage : Institution of Electrical Engineers, pp. 153-4..

[2] Vectornav 2011, Inertial Navigation, viewed Oct 03 2011, <http://www.vectornav.com/index.php?option=com_content\&view=arti cle\&id=27\&Itemid $=11>$.

[3] Liu, H. \& Pang, G. 1999, 'Accelerometer for mobile robot positioning', Industry Applications Conference, 1999. Thirty-Fourth IAS Annual Meeting. Conference Record of the 1999 IEEE, vol. 3, pp. 1735-42 vol.3.

[4] Barshan, B. \& Durrant-Whyte, H.F. 1995, 'Inertial navigation systems for mobile robots', Robotics and Automation, IEEE Transactions on, vol. 11 , no. 3, pp. 328-42.

[5] Titterton, D.H.D.H. 2004b, 'Accelerometer and multi-sensor technology', in D. Titterton \& Weston, Eds., Strapdown Inertial Navigation Technology, 2nd edn., vol. 5, Stevenage : Institution of Electrical Engineers., pp. 156-9

[6] Lueck, R. and Wolk, F. 2002, 'A quick method for calibrating accelerometers', research paper, Rockland Scientific International Inc., April.

[7] Wahyudi and Ngatelan, "Design of multi-sensor IMU for land vehicle," 2015 2nd International Conference on Information Technology, Computer, and Electrical Engineering (ICITACEE), Semarang, 2015, pp 271-274.

[8] L. Tao and D. Hongwang, "A camera-IMU system extrinsic parameter calibration method," 2017 IEEE 2nd Information Technology, Networking, Electronic and Automation Control Conference (ITNEC), Chengdu, 2017, pp. 1063-1066.

[9] Y. Chen, G. Zhang, J. Guo, R. Mehmood and Y. Liu, "A Separable Digital Protractor Based on IMU for Angle Measurement," 2016 International Conference on Identification, Information and Knowledge in the Internet of Things (IIKI), Beijing, 2016, pp. 227-231.

[10] H. Benzerrouk and A. V. Nebylov, "Robust IMU/UWB integration for indoor pedestrian navigation," 2018 25th Saint Petersburg International Conference on Integrated Navigation Systems (ICINS), St. Petersburg, 2018, pp. $1-5$ 